\title{
Wnt/Planar Cell Polarity Signaling Controls the Anterior-Posterior Organization of Monoaminergic Axons in the Brainstem
}

\author{
Ali G. Fenstermaker, ${ }^{1 *}$ Asheeta A. Prasad, ${ }^{2 *}$ Ahmad Bechara, ${ }^{1}$ Youri Adolfs, ${ }^{2}$ Fadel Tissir, ${ }^{3}$ Andre Goffinet, ${ }^{3}$ \\ Yimin Zou, ${ }^{1}$ and R. Jeroen Pasterkamp ${ }^{2}$ \\ ${ }^{1}$ Neurobiology Section, Biological Science Division, University of California, San Diego, La Jolla, California 92093, ${ }^{2}$ Department of Neuroscience and \\ Pharmacology, Rudolf Magnus Institute of Neuroscience, University Medical Center Utrecht, 3584 CG Utrecht, The Netherlands, and ${ }^{3}$ Université Catholique \\ de Louvain, Institute of Neuroscience, B-1200 Brussels, Belgium
}

\begin{abstract}
Monoaminergic neurons [serotonergic (5-HT) and dopaminergic (mdDA)] in the brainstem project axons along the anterior-posterior axis. Despite their important physiological functions and implication in disease, the molecular mechanisms that dictate the formation of these projections along the anterior-posterior axis remain unknown. Here we reveal a novel requirement for Wnt/planar cell polarity signaling in the anterior-posterior organization of the monoaminergic system. We find that 5-HT and mdDA axons express the core planar cell polarity components Frizzled3, Celsr3, and Vangl2. In addition, monoaminergic projections show anterior-posterior guidance defects in Frizzled3, Celsr3, and Vangl2 mutant mice. The only known ligands for planar cell polarity signaling are Wnt proteins. In culture, Wnt5a attracts 5-HT but repels mdDA axons, and Wnt7b attracts mdDA axons. However, mdDA axons from Frizzled3 mutant mice are unresponsive to Wnt5a and Wnt7b. Both Wnts are expressed in gradients along the anterior-posterior axis, consistent with their role as directional cues. Finally, Wnt $5 a$ mutants show transient anterior-posterior guidance defects in mdDA projections. Furthermore, we observe during development that the cell bodies of migrating descending 5-HT neurons eventually reorient along the direction of their axons. In Frizzled 3 mutants, many 5-HT and mdDA neuron cell bodies are oriented abnormally along the direction of their aberrant axon projections. Overall, our data suggest that Wnt/planar cell polarity signaling may be a global anterior-posterior guidance mechanism that controls axonal and cellular organization beyond the spinal cord.
\end{abstract}

\section{Introduction}

Axon guidance cues control the direction of growth cone navigation along the major anatomical axes [anterior-posterior (A-P), dorsal-ventral, and inferior-superior] during neural circuit development. Wnt family proteins are evolutionary conserved A-P guidance cues (Zou, 2006; Zou and Lyuksyutova, 2007). In the vertebrate spinal cord, Wnts provide directional cues for both ascending sensory axons and descending motor axons (Lyuksyutova et al., 2003; Liu et al., 2005; Wolf et al., 2008). In contrast, the A-P

\footnotetext{
Received Aug. 28, 2010; accepted 0ct. 2, 2010.

This work is supported by National Institutes of Health Grant R01 NS047484 (Y.Z.) and by the Netherlands Organization for Health Research and Development (ZonMW-VIDI and ZonMW-TOP), the International Parkinson Foundation, the Human Frontier Science Program (Career Development Awards), and the European Union (mdDANeurodev, FP7/2007-2011, Grant 222999) (R.J.P.). This study was partly performed within the framework of Dutch Top Institute Pharma Project T5-207 (R.J.P.). We are grateful to Tony Wynshaw Boris for sharing the Looptail mice, Steven Stacker for providing the Ryk mutant mice, and Francesca Morello and Katsumi Fumoto for help with immunohistochemistry and confocal microscopy. We thank Rejji Kuruvilla and Jeremy Nathans for generously providing the Fzd3 mutant mice.

*A.G.F. and A.A.P. contributed equally to this work

Correspondence should be addressed to either of the following: Yimin Zou, Neurobiology Section, Biological Science Division, University of California, San Diego, La Jolla, CA 92093, E-mail: yzou@ucsd.edu; or Jeroen Pasterkamp, Department of Neuroscience and Pharmacology, Rudolf Magnus Institute of Neuroscience, University Medical Center Utrecht, 3584 CG Utrecht, The Netherlands, E-mail: r.j.pasterkamp@umcutrecht.nl.

DOI:10.1523/JNEUROSCI.4508-10.2010

Copyright $\odot 2010$ the authors $\quad 0270-6474 / 10 / 3016053-12 \$ 15.00 / 0$
}

axon guidance cues that function in the vertebrate CNS more rostral to the spinal cord remain essentially unknown.

Monoaminergic neurons [serotonergic (5-HT) and dopaminergic (mdDA)] in the brainstem control many aspects of nervous system functioning, and their malfunctions are involved in the pathogenesis of a large number of nervous system diseases, such as depression, autism, and Parkinson's disease (Pardo and Eberhart, 2007; Van den Heuvel and Pasterkamp, 2008). These neurons are organized in discrete nuclei in the midbrain (mdDA; A9, A10) and hindbrain (5-HT; B1-B9) and project axons along the A-P axis to reach their final targets in the brain and spinal cord (Rubenstein, 1998; Goridis and Rohrer, 2002; Cordes, 2005; Smidt and Burbach, 2007; Van den Heuvel and Pasterkamp, 2008). The A-P projections of 5-HT and mdDA neurons are established during midgestation and are essential for appropriate monoaminergic circuit formation during subsequent developmental stages. Surprisingly little is known about the genetic control of monoaminergic pathway development, particularly how monoaminergic projections are initially established along the A-P axis.

One of the Wnt signaling pathways, the planar cell polarity (PCP) pathway, is involved in tissue morphogenesis and directed cell migration (Wang and Nathans, 2007; Zallen, 2007; Goodrich, 2008; Simons and Mlodzik, 2008). Core PCP components include Frizzled, Flamingo (Celsrs in vertebrates), Van Gogh, 
A
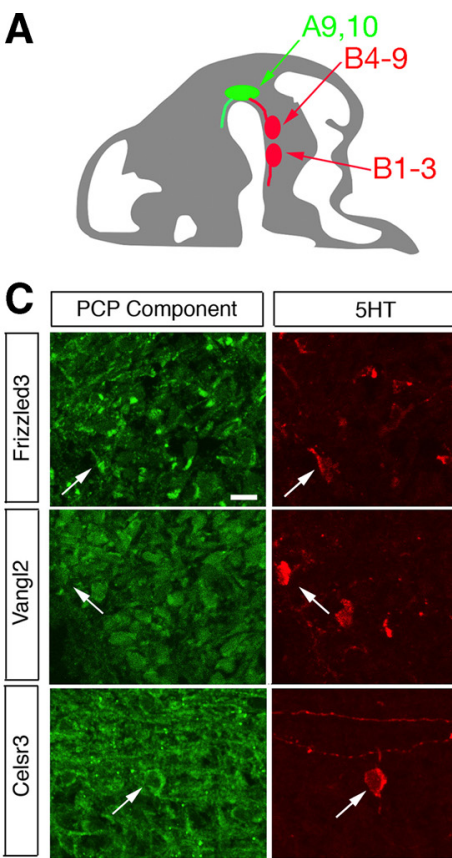

D

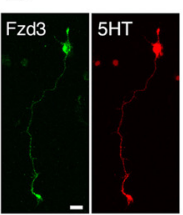

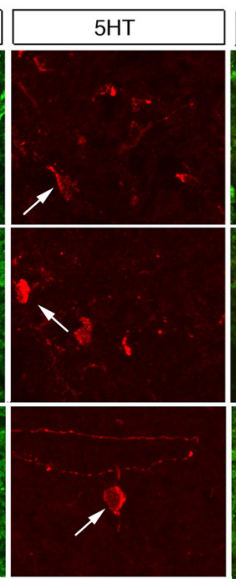

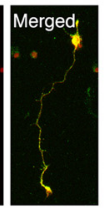

$E$

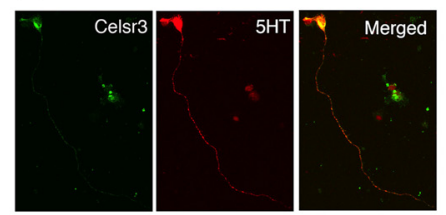

B
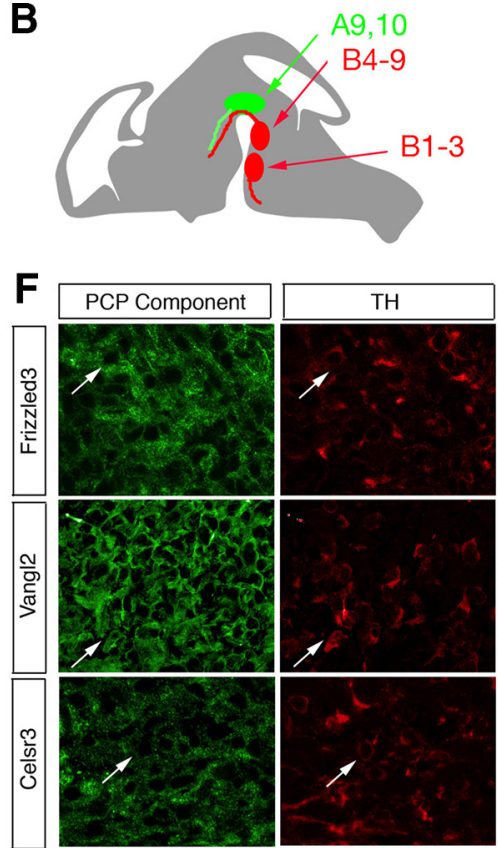

G

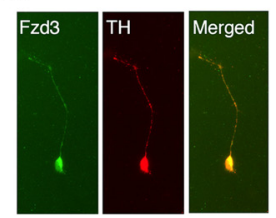

H

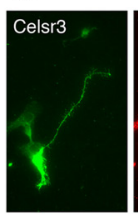

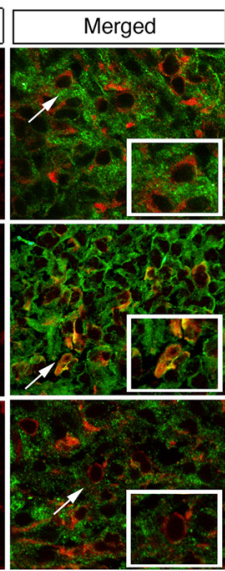

E14.5

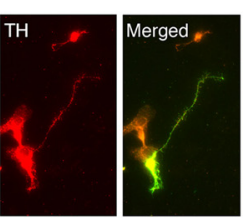

Figure 1. PCP signaling components are expressed in 5 -HT- and TH-positive neurons during midbrain and hindbrain development. Schematics in $\boldsymbol{A}$ and $\boldsymbol{B}$ indicate the anatomical location of the descending and ascending serotonin systems (B1-B3 and B4-B9; red) and the mesodiencephalic dopamine system (A9, A10; green) in mouse at E12.5 and E14.5, respectively. C, Immunohistochemistry for 5-HT in red and PCP receptors in green in sagittal sections through the embryonic hindbrain at E12.5. Frizzled3, Vangl2, and Celsr3 all colocalize with 5-HT-positive neurons and fibers. Insets depict higher-magnification images of cells indicated by the arrow. $D$, Confocal images showing immunohistochemistry for TH in red and PCP receptors in green in coronal sections through the E14.5 midbrain. Frizzled3, VangI2, and Celsr3 all colocalize with TH-positive neurons and fibers. Insets depict higher-magnification images of cells indicated by the arrow. $\boldsymbol{D}, \boldsymbol{E}, 5$-HT-positive dissociated neurons coimmunostained with 5-HT in red and Fzd3 $(\boldsymbol{D})$ or Celsr3 $(\boldsymbol{E})$ in green. $\boldsymbol{G}, \boldsymbol{H}$, TH-positive dissociated neurons coimmunostained with TH in red and Fzd3 $(\boldsymbol{G})$ or $(\mathrm{Celsr} 3(\boldsymbol{H})$ in green. Scale bars, $20 \mu \mathrm{m}$.

Disheveled, Prickle, and Diego. In this study, we found that Wnt/ PCP signaling is essential for the A-P organization of 5-HT and mdDA axons in the brainstem. Both 5-HT and mdDA neurons expressed core PCP components and showed A-P guidance defects in Frizzled3 (Fzd3), Celsr3, and Vangl2 [Looptail (Lp)] mutant mice. In addition, Wnt5a mutants showed transient A-P guidance defects for mdDA axons. Wnt5a and $W n t 7 b$ were expressed in gradients along the $\mathrm{A}-\mathrm{P}$ axis in the brainstem, consistent with their role as directional cues. In culture, Wnt5a attracted 5-HT axons but repelled mdDA axons, whereas Wnt7b attracted mdDA axons. In contrast, dopaminergic neurons derived from Fzd3 mutant mice did not respond to Wnt5a or Wnt7b. Analyses of wild-type or Fzd3 mutant mice further revealed that cell bodies of descending 5-HT neurons are initially oriented mediolaterally and gradually shift to orient along the A-P axis following the direction of their axons and that proper Wnt/PCP signaling is essential for this A-P cellular organization of monoaminergic nuclei. Together, these results define novel and crucial roles for Wnt/PCP signaling in establishing the A-P axonal and cellular organization of monoaminergic systems. Also, these findings indicate that $\mathrm{A}-\mathrm{P}$ axon guidance functions of different PCP proteins are not limited to the spinal cord but function more globally in the vertebrate CNS.

\section{Materials and Methods}

Animals. All animal use and care were in accordance with institutional guidelines. CD-1 and C57BL/6 mice were obtained from Charles River. Fzd3 mutant mice were obtained from Jeremy Nathans [Johns Hopkins University School of Medicine, Baltimore, MD (Wang et al., 2002)], Looptail mice were from Tony Wynshaw Boris [University of California, San Francisco, San Francisco, CA (Montcouquiol et al., 2006)], and Ryk mutant mice were from Steven Stacker [Ludwig Institute for Cancer Research, Melbourne, VIC, Australia (Halford et al., 2000)]. Celsr3 mutant mice have been described previously (Tissir et al., 2005). Wnt5a mutant mice were purchased from The Jackson Laboratory. Timed-pregnant mice were killed by means of cervical dislocation. The morning on which a vaginal plug was detected was considered embryonic day 0.5 (E0.5). For in situ hybridization and immunohistochemistry, E11.5-E17.5 brains were fixed in 4\% paraformaldehyde (PFA) for $30 \mathrm{~min}$ to $1 \mathrm{~h}$, followed by immersion in $30 \%$ sucrose. Brains were frozen on powdered dry ice, cryosectioned, mounted on Superfrost Plus slides (Thermo Fisher Scientific), air dried, and stored desiccated at $-80^{\circ} \mathrm{C}$.

In situ hybridization and immunohistochemistry. Nonradioactive in situ hybridization on cryosections (14 $\mu \mathrm{m})$ was performed as described previously (Pasterkamp et al., 2007). Specific digoxigenin-labeled in situ probes for the mouse Wnt family were as described (Liu et al., 2005). Whole-mount in situ hybridization was preformed as described previously (Henrique et al., 1995).

Cryosections $(14 \mu \mathrm{m})$ were stained immunohistochemically as described previously (Kolk et al., 2009). Sections were counterstained with 

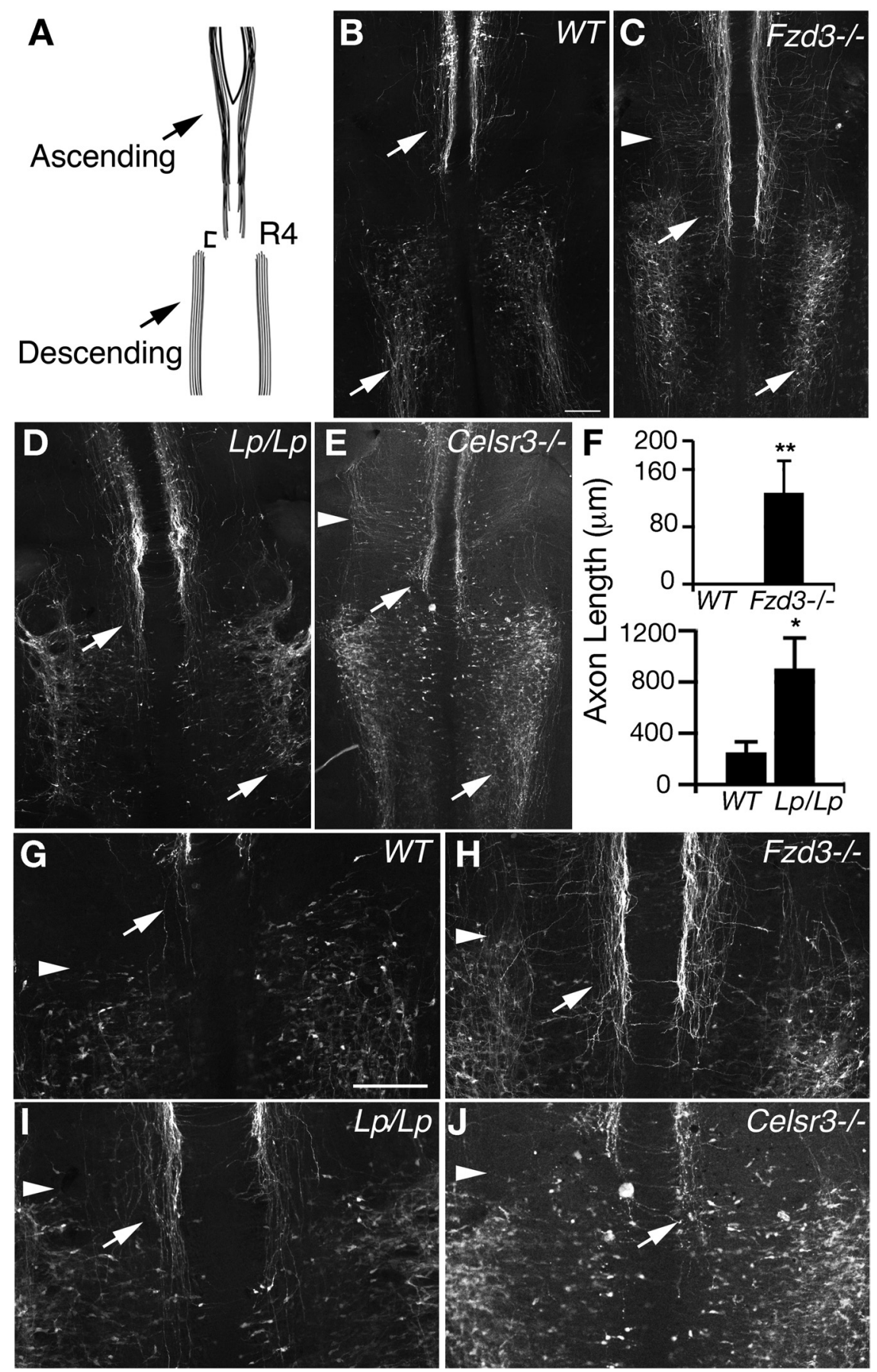

Figure 2. $P C P$ signaling is required for axonal pathfinding of hindbrain serotonergic neurons. $A$, Schematic representation of the developing 5-HT system atE12.5. B-E, Whole-mount immunostaining against 5-HT in the hindbrain. AtE12.5, ascending 5-HT neurons in $F z d 3^{-1-}(\boldsymbol{C}), L p / L p(\boldsymbol{D})$, and $C e l s r 3^{-/-}(\boldsymbol{E})$ mice all display axon misprojections into R4 (see top arrows). Fzd $3^{-1-}$ and Celsr $3^{-1-}$ also display laterally projecting axons within the ascending population (arrowheads in $C, E$ ). In addition, $\mathrm{Fzd}^{-1-}(\boldsymbol{C})$ and $L p / L p(\boldsymbol{D})$ mice display aberrant anteriorly projecting 5 -HT projections in the descending population and a marked reduction of proper descending axons (bottom arrows). $\boldsymbol{F}$, Length of axon invasion into R4 in the $F z d 3^{-1-}$ and $L p / L p$ mouse. $\mathbf{G}-J$, Higher magnification of premature R4 invasion. Arrowheads indicate the start of the descending population, and the arrow label axons from the ascending population. Scale bar, $250 \mu \mathrm{m}$. WT, Wild type. ${ }^{* *} p<0.005,{ }^{*} p<0.03$.

fluorescent Nissl (1:500; Neurotrace, Invitrogen) or 4',6' -diamidino-2phenylindole (DAPI) (Sigma), washed extensively, and embedded in Mowiol (Sigma). Staining was visualized using a Carl Zeiss Axioskop 2 microscope or by confocal laser-scanning microscopy (LSM510; Carl Zeiss). The following primary antibodies were used: rabbit anti-Frizzled3 (1:500; a gift from Jeremy Nathans), rabbit anti-Celsr3 (1:500; generated in the Zou laboratory), goat anti-Vangl2 (1:100; Santa Cruz Biotechnology), rat anti-
5-HT (1:500; Accurate Chemicals), rabbit antityrosine hydroxylase (TH) (1:1000; Pel-Freeze), and mouse anti-TH (1:500; Immunostar).

TH explant assays. Three-dimensional collagen matrix assays using substantia nigra (SN) and ventral tegmental area (VTA) explants were performed as described previously (Kolk et al., 2009). SN or VTA explants were rapidly dissected from E12.5-E14.5 C57BL/6 embryos or from wild-type or Frizzled 3 mutant embryos and embedded in close proximity to $(\sim 300$ $\mu \mathrm{m}$ apart) aggregates of HEK293 cells transiently transfected with expression vectors for Wnt5a or Wnt7b, or mock-transfected as a control. Explants were cultured for $56-58 \mathrm{~h}$, fixed, and coimmunostained using rabbit anti-TH (1:1000; Pel-Freeze) and mouse anti$\beta$ III-tubulin (1:3000; Covance) antibodies to stain dopaminergic and all neurites emerging from the explants, respectively.

For quantification of explant assays, the length of the 20 longest TH-positive neurites was measured in both the proximal and distal quadrants of the explant cultures using OpenLab software (Improvision). The average value of the 20 neurites proximal and distal was used to determine the proximal/distal ratio (P/D ratio) per explant. Data were statistically analyzed by one-way ANOVA $(\alpha=5 \%)$ and expressed as means \pm SEM.

5-HT open-book explants. Hindbrains were dissected from E12.5 time pregnant CD-1 mice in L15 media and placed ventricular zone down onto a bed of collagen. Blue Sepharose beads previously coated overnight at $4^{\circ} \mathrm{C}$ in $100 \mathrm{ng} / \mu \mathrm{l}$ purified Wnt proteins were placed along the midline of the explants, and then the explant was covered in a top layer of collagen. Explants were incubated at $37^{\circ} \mathrm{C}$ for $24 \mathrm{~h}$ and fixed with $4 \%$ PFA overnight at $4^{\circ} \mathrm{C}$. 5 -HT-positive neurons were visualized using whole-mount immunostaining with rat anti-5-HT (1:300; Accurate Chemicals). Images were acquired by confocal microscopy, and $Z$-stacks were compressed and analyzed in NIH ImageJ.

For the quantification of hindbrain openbook cultures, we counted the number of 5-HTpositive axons emanating from the midline of the ascending population. The axons were grouped as growing anteriorly, laterally, or posteriorly. The number of axons growing anteriorly was graphed as a percentage of the total number of axons counted. More than 100 axons were counted for each explant (control, $n=10$; Wnt4, $n=6$; Wnt5a, $n=10$; Wnt7a, $n=9$ ). Data were statistically analyzed by one-tailed Student's $t$ test and represented as \pm SEM.

Quantification of premature axon invasion into rhombomere 4. For quantification of the axon guidance defect of the ascending 5-HT population, we measured the length of axons past the start of the descending neuron population (see Fig. 2G-J, arrowhead). Both Fzd3 and $L p$ mice were compared with littermate controls because there were developmental differences among different genetic backgrounds (B6 mixed vs Sv129J mixed). This analysis was used to show that the mutants invaded the region below rhombomere 4 (R4) before their wild-type counterparts did. $\left(F z d 3^{+/+}, n=6, F z d 3^{-/-}, n=4 ; L p^{+/+}, n=4 ; L p / L p, n=4\right)$. Data were statistically analyzed by one-tailed Student's $t$ test and represented as \pm SEM. 
Dissociated neuron cultures. To generate dissociated neuron cultures from the midbrain, the mdDA system was microdissected from E14.5 C57BL/6 wild-type embryos. Tissue from several different embryos was pooled and incubated in trypsin for $20 \mathrm{~min}$ at $37^{\circ} \mathrm{C}$. Trypsin activity was inhibited with $20 \%$ fetal calf serum. Then, the cells were pelleted by mild centrifugation and resuspended in Neurobasal medium supplemented with B27, penicillin/streptomycin, and glutamine. A single-cell suspension was generated by putting the cells through a $70 \mu \mathrm{m}$ filter after which cells were plated on poly-D-lysineand laminin-coated glass coverslips in a 12-well plate for $2-3 \mathrm{~d}$. Hindbrain neuron cultures were generated similar to dopaminergic neurons but with the following modifications. Hindbrains were dissected anterior to rhombomere 4 from E12.5 CD1 mouse embryos. Tissue from multiple embryos was pooled in incubated in $0.025 \%$ trypsin for $20 \mathrm{~min}$ at $37^{\circ} \mathrm{C}$. The tissue was then triturated with fire polished glass pasture pipettes, and trypsin activity was inhibited by the addition of RPMI plus $5 \%$ horse serum. Cells were plated in Neurobasal media as above on poly-D-lysine- and laminin-coated glass coverslips and incubated for $2 \mathrm{~d}$. All cells were fixed with $4 \%$ paraformaldehyde for $10 \mathrm{~min}$ at room temperature and immunostained with the following primary antibodies: rabbit antiFrizzled3 (1:500; a gift from Jeremy Nathans), rabbit anti-Celsr3 (1:500), goat anti-Vangl2 (1: 100; Santa Cruz Biotechnologies), rat anti5-HT (1:500; Accurate Chemicals), rabbit anti-TH (1:1000; Pel-Freeze), and mouse anti-TH (1:500; Immunostar).

Quantification of cell body orientation. To assess and quantify mdDA cell body orientation defects in $F z d 3$ mutant mice, sagittal sections from E12.5 $\mathrm{Fzd}^{-/-}$embryos $(n=3)$ and littermate controls $(n=3)$ were immunostained with anti-TH antibodies and counterstained with DAPI. Images through the lateral and medial midbrain region were captured from these sections at identical medial to lateral locations using a Carl Zeiss Axioskop 2 microscope. Then, using Carl Zeiss Axiovision software, angles were determined between the lining of the mesencephalic flexure (baseline, represents $0^{\circ}$ ) and the trajectory of the initial axon segment just proximal to the TH-positive cell body. Fifty to 100 TH-positive neurons were measured per embryo at the lateral and medial levels, and angles were grouped $\left(0 / 45^{\circ}, 45 / 90^{\circ}\right.$, etc.) and calculated as percentage of total.

To analyze the orientation of 5-HT cell bodies of the descending population, we imaged whole-mount immunostaining of 5-HT neurons using anti-5-HT antibodies from E12.5 $\mathrm{Fzd}^{-1-}(n=3)$ and wild-type littermate controls $(n=3)$. $Z$-stack images were acquired by confocal microscopy and analyzed with NIH Image J. Cell body orientation was assessed by drawing a line vertically down to the center of the cell, followed by drawing an intersecting line through the center of the initial segment of the leading process. The angle of this intersection was measured in NIH Image J. Using this method, a completely horizontal cell would give an angle of $90^{\circ}$, whereas a completely vertical cell would yield an angle of $180^{\circ}$. More than 1005 -HT-positive cells were counted per embryo, and the angles were binned $\left(0 / 45^{\circ}, 45 / 90^{\circ}\right.$, etc. $)$ and graphed $L p / L p, n=4$ for E12.5.
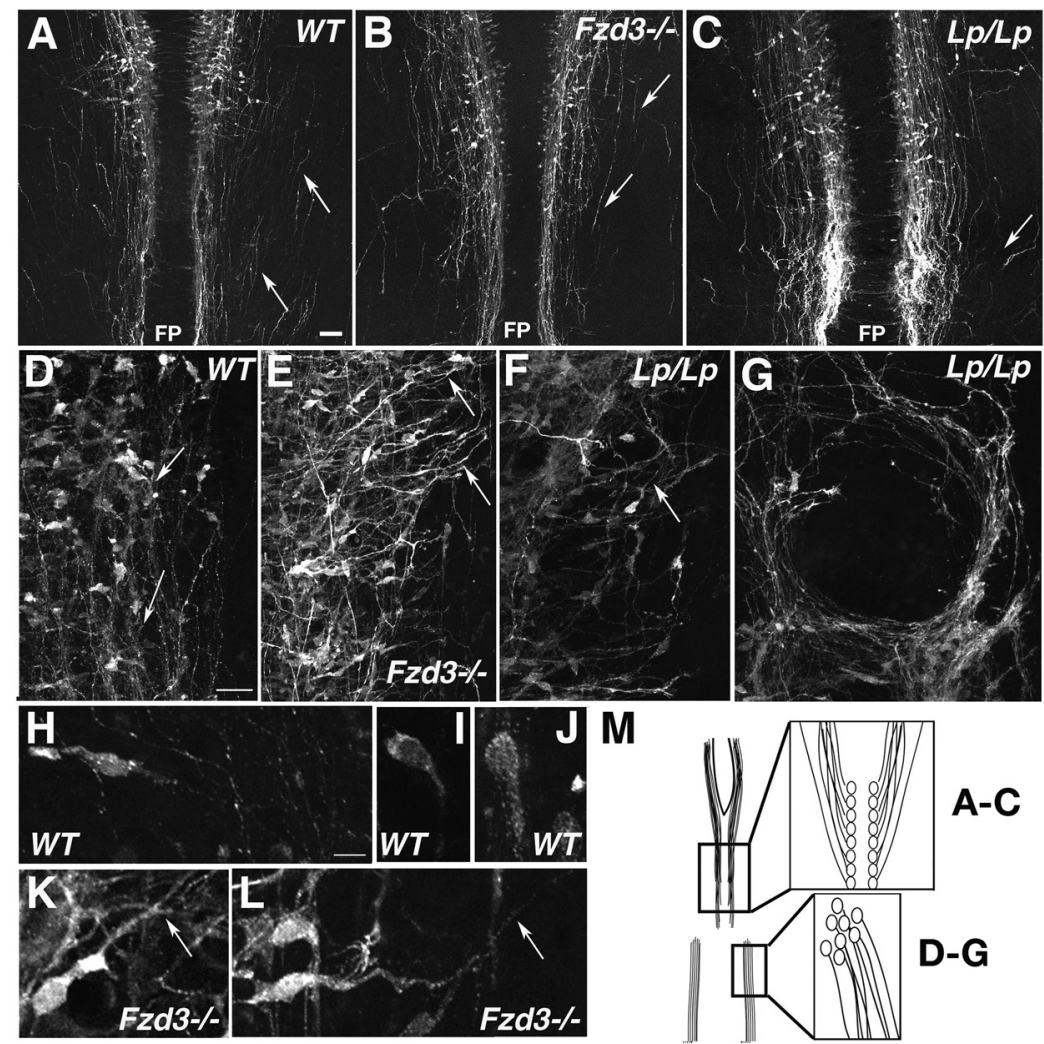

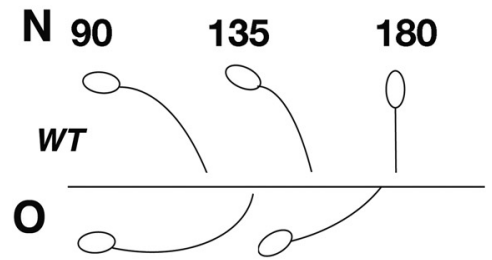

Fzd3-/

$\mathrm{M}$

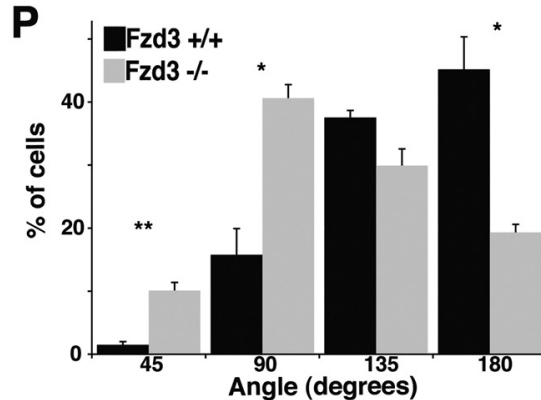

Figure 3. PCP mutant mice display specific axon guidance and cell body orientation defects in the 5 -HT system. $A-L$, Whole-mount 5-HT immunostaining. Ascending 5-HT populations are depicted in A-C, and descending 5-HT axons are shown in D-L. This is schematized in $\boldsymbol{M}$. $\boldsymbol{A}$, In E12.5 wild-type (WT) embryos, axons in the ascending 5-HT population emanate from the floor plate (FP) and strictly grow anterior toward the brain (arrows). In contrast, $F_{z d 3^{-/-}}(\boldsymbol{B})$ or $L p / L p(C)$ mice display ascending axons that grow laterally and posteriorly (arrows). Lp/Lp mice display many "wavy" axons characteristic of axons lacking appropriate guidance. $\boldsymbol{D}$, In wild-type mice, descending axons project posteriorly toward the spinal cord at $E 12.5$ (arrows). Both $F z d 3^{-\prime-}(E)$ and $L p / L p(F, G)$ mice display aberrant descending projections (arrows). $F z d 3^{-1-}$ mice form very short axons within the descending region, many of which project into inappropriate lateral and anterior directions $(\boldsymbol{E})$. $G, L p / L p$ mice form axons within the descending region that display clear fasciculation defects and grow in large circles. Cell body orientation is also affected in $\mathrm{Fzd}^{-/}{ }^{-}$mice. Although during their mediolateral migration most wild-type descending neurons display cell bodies oriented first laterally (en route to theirfinal position) and then along the A-Paxis as they reach theirfinal lateral position $(\boldsymbol{H}-\boldsymbol{J})$, cell bodies in $\mathrm{Fzd}^{-/-}$mice are oriented in several different directions, many projecting at an acute angle near the midline and failing to orient posteriorly as they reach their lateral resting position $(K, L)$. The cell orientation phenotype is schematized in $N$ and $\boldsymbol{O}$ and quantified in $\boldsymbol{P}$. Graph shows average \pm SEM. Almost $50 \%$ of $F z d 3^{+/+}$mice are oriented at $180^{\circ}$ compared with $F z d 3^{-/-}$mice in which only $\sim 10 \%$ of neurons are oriented posteriorly.M, Medial; L, lateral. Scale bars: $A-C, 50 \mu \mathrm{m} ; \mathbf{D}-\mathbf{G}, 20 \mu \mathrm{m} .{ }^{*} p<0.05,{ }^{* *} p<0.01$. Number of mutants analyzed: Fzd3 ${ }^{-\prime-} ; n=3$ forE11.5, $n=15$ forE12.5; $n=2$ for E13.5; Celsr3 ${ }^{-\prime-}, n=3$ for E11.5; $n=4$ for E12.5;

as percentage of total as for TH quantification. Data were statistically analyzed by one-tailed Student's $t$ test and represented as \pm SEM.

\section{Results}

Serotonergic and dopaminergic neurons express core PCP components

In mouse, ascending 5-HT neurons are born between E11.5 and E12.5, just posterior to the isthmus, and are located close to the 

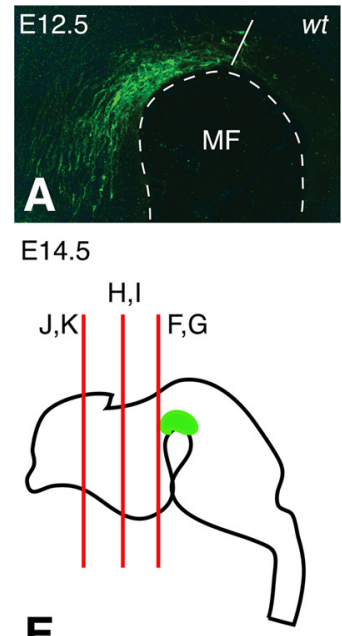

E
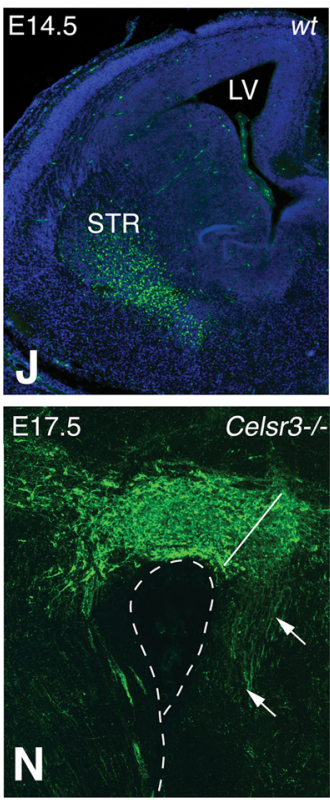
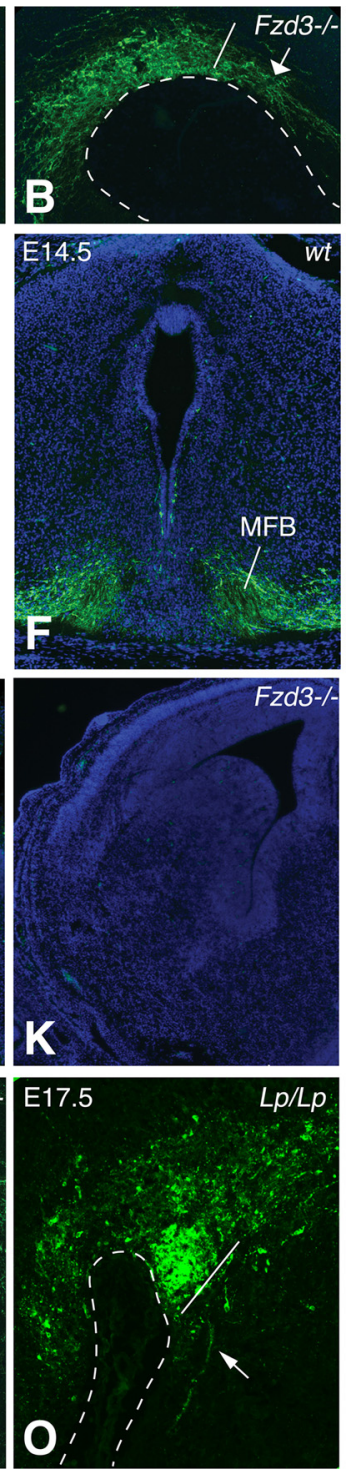
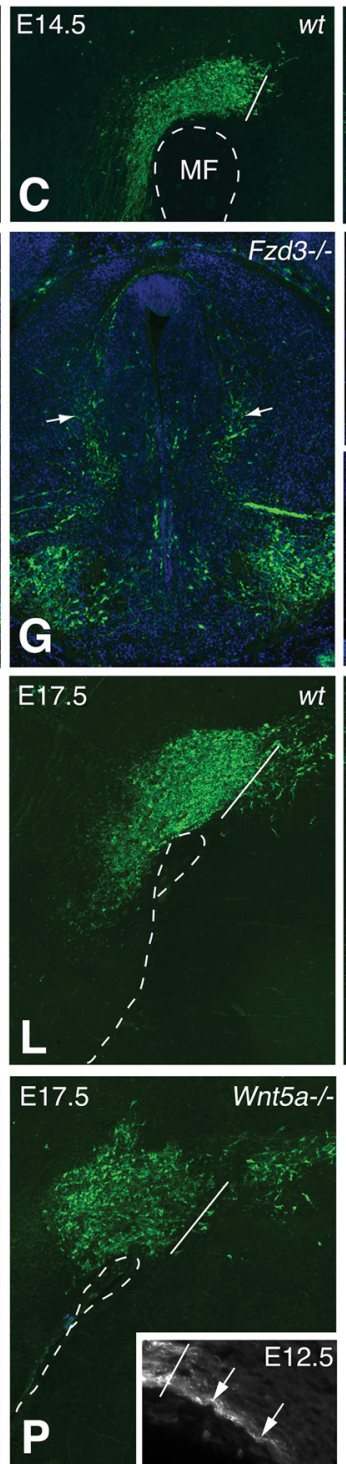
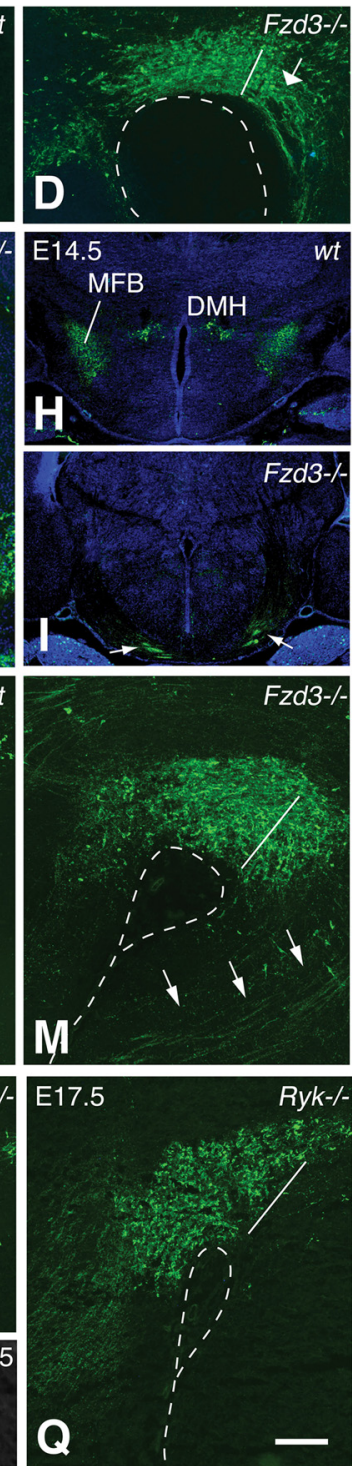

Figure 4. PCP mutant mice display axon guidance defects in the mdDA system. Immunohistochemistry for TH (in green) was used to analyze mdDA axon projections in Fzd $3^{-/-}$, Celsr $^{-/-}$, $L p / L p, W n t 5 a^{-/-}$, and $R y k^{-/-}$mice and littermate controls at E12.5 $(\boldsymbol{A}, \boldsymbol{B}), \mathrm{E} 14.5(\boldsymbol{C}-\boldsymbol{K})$, and E17.5 $(\boldsymbol{L}-\boldsymbol{Q})$. Coronal sections shown in $\boldsymbol{F}-\boldsymbol{K}$ are counterstained with fluorescent Nissl to demarcate anatomical features. $\boldsymbol{A}-\boldsymbol{D}$ and $\boldsymbol{L}-\mathbf{Q}$ show sagittal sections. Whereas in wild-type (wt) control mice mdDA neurons project their axons anteriorly toward the forebrain, numerous TH-positive axons projecting posteriorly into the hindbrain region are found in $F z d 3^{-/-}$mice at E12.5, E14.5, and E17.5 (A-D, $\boldsymbol{L}, \boldsymbol{M}$, arrows indicate posterior misprojections). Dotted line outlines mesencephalic flexure (MF) and white line the posterior boundary of the mdDA system. Schematic in $\boldsymbol{E}$ indicates the location of sections shown in $\boldsymbol{F}-\boldsymbol{K}$. The medial forebrain bundle (MFB) is severely disrupted in $\mathrm{Fzd}^{-/-}$mice, and ectopic axons can be found at dorsal levels in the brainstem but also near the midline $(\boldsymbol{F}, \boldsymbol{G})$ and in abnormal ventral regions in the developing hypothalamus (DMH) near the optic chiasm $(\boldsymbol{H}, \boldsymbol{I})$. In addition, TH-positive axons fail to reach their synaptic targets such as the striatum $(\boldsymbol{J}, \boldsymbol{K})$ in Fzd $3^{-/-}$mice. Celsr $3^{-/-}$mice show a massive posterior backprojection of TH-positive

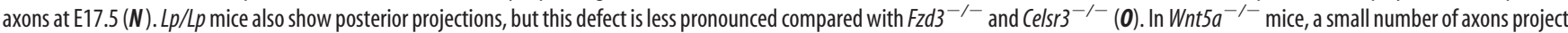
posteriorly at E12.5 (inset) (supplemental Fig. S4, available at www.jneurosci.org as supplemental material), but at E17.5 this aberrant projection is no longer observed (P). Ryk ${ }^{-/-}$mice show normal TH-positive axon projections (Q). Number of mutants analyzed:Fzd3 ${ }^{-/-}, n=13$ forE12.5; $n=19$ forE14.5; $n=8$ forE17.5; Celsr3 ${ }^{-/-}, n=4$ forE17.5; $L p / L p, n=2$ forE17.5; Wnt5a ${ }^{-/-}$, $n=4$ for E17.5; Ryk ${ }^{-/-}, n=3$ for E17.5. Scale bar: $\boldsymbol{A}-\boldsymbol{D}, \boldsymbol{F}-\boldsymbol{K}, 100 \mu \mathrm{m} ; \boldsymbol{L}-\mathbf{Q}, 50 \mu \mathrm{m}$.

midline (Fig. $1 A, B$, top red oval). They reside in six different nuclei (B4-B9) and send projections anteriorly to innervate various CNS structures. Shortly after the appearance of the ascending 5-HT neurons, descending 5-HT neurons migrate laterally in rhombomeres 5-7, become immunoreactive for 5-HT, and begin projecting axons posteriorly by E12.5 (Fig. $1 A, B$, bottom red oval). These neurons reside in three distinct nuclei (B1-B3) and project axons into the cervical spinal cord. Despite the well characterized anatomy of the 5-HT system, its axon guidance mechanisms, particularly along the A-P axis, are unknown. The first mdDA neurons, located just anterior to the isthmus, are born at approximately E10.5 and start to extend axons at E11.5. The
mdDA system is composed of a medial population close to the midline, the VTA (A10), and a lateral subdivision, the SN (A9) (Fig. $1 A, B$, green oval), and both give rise to prominent ascending (anterior) axonal projections to the striatum and the medial prefrontal cortex.

It is evident that robust mechanisms must be in place to guide both hindbrain 5-HT and midbrain mdDA axons into their normal A-P directions. Wnts and their receptors have been implicated in determining the growth polarity of long descending and ascending spinal axon projections (Lyuksyutova et al., 2003; Liu et al., 2005; Wolf et al., 2008). In addition, Wnts and their receptors are expressed in the midbrain during early embryonic devel- 
opment to regulate neuronal proliferation and differentiation (Andersson et al., 2008). Furthermore, in mice lacking Frizzled3, dopaminergic innervation of the striatum is lost (Wang et al., 2002). These results suggest that Fzd3 and associated signaling proteins may act to guide the growth of monoaminergic axons.

To test this hypothesis, we first examined the expression of core PCP components in the developing hindbrain by coimmunostaining against PCP proteins and 5-HT. We examined sagittal sections of the mouse hindbrain at E11.5 (supplemental Fig. S1, available at www.jneurosci.org as supplemental material) and E12.5, stages at which 5-HT neurons are born and begin pathfinding. We found that several PCP core components, Fzd3, Vangl2, and Celsr3, are expressed at E11.5 and E12.5 in both the ascending and descending 5-HT populations in cell bodies and fibers (Fig. 1C). Most, if not all, 5-HT-positive cells expressed Fzd3, Vangl2, and Celsr3. We also performed dissociated neuron cultures followed by immunocytochemistry and found that all 5-HT-positive cells express Fzd3 (Fig. 1D) and Celsr3 (Fig. 1E). To examine the expression of core PCP components in mdDA neurons, we used coimmunostaining against PCP proteins and $\mathrm{TH}$ on coronal sections. We focused on E11.5-E14.5, during which mdDA axon growth polarity is established. Fzd3, Celsr3, and Vangl2 are widely expressed in the midbrain region and in all TH-positive mdDA neurons as exemplified by E14.5 (Fig. 1F). Using dissociated neuron culture and immunocytochemistry, we confirmed that all TH-positive cells express Fzd3 and Ceslr3 (Fig. 1G,H). Unfortunately, the Vangl2 antibody did not stain well in the dissociated cultures. However, the immunostaining in Figure 1, $C$ and $F$, clearly shows the presence of Vangl2 in 5-HT and TH neurons.

\section{PCP signaling is required for A-P guidance of serotonergic axons}

To examine the potential role of PCP signaling in the development of the 5-HT system, we analyzed mouse lines deficient in PCP signaling components (Figs. 2, 3). Whole-mount immunostaining against 5-HT in the hindbrain reveals both the cell bodies and axons of serotonergic neurons (Figs. 2, 3). The Fzd3 $3^{-1-}$ mouse (Wang and Nathans, 2007) displays dramatic axon guidance defects in the 5-HT system at E12.5 (Fig. 2A-C). Although 5-HT-positive cell bodies appear to be in the appropriate A-P positions in $\mathrm{Fzd}^{-/-}$mice, the axons in both the ascending and descending populations display marked misprojections. Axons in the ascending population project posteriorly and laterally instead of strictly anteriorly (Figs. $2 C, H, 3 B$ ). At E12.5, when there are normally no axons present in $\mathrm{R} 4$, axons erroneously descend well into this rhombomere (Fig. 2C,F,H). The descending 5-HT population also displays striking axon guidance defects. Descending axons are distinctly shorter than their wild-type counterparts and project randomly along the A-P axis (Fig. 2C).
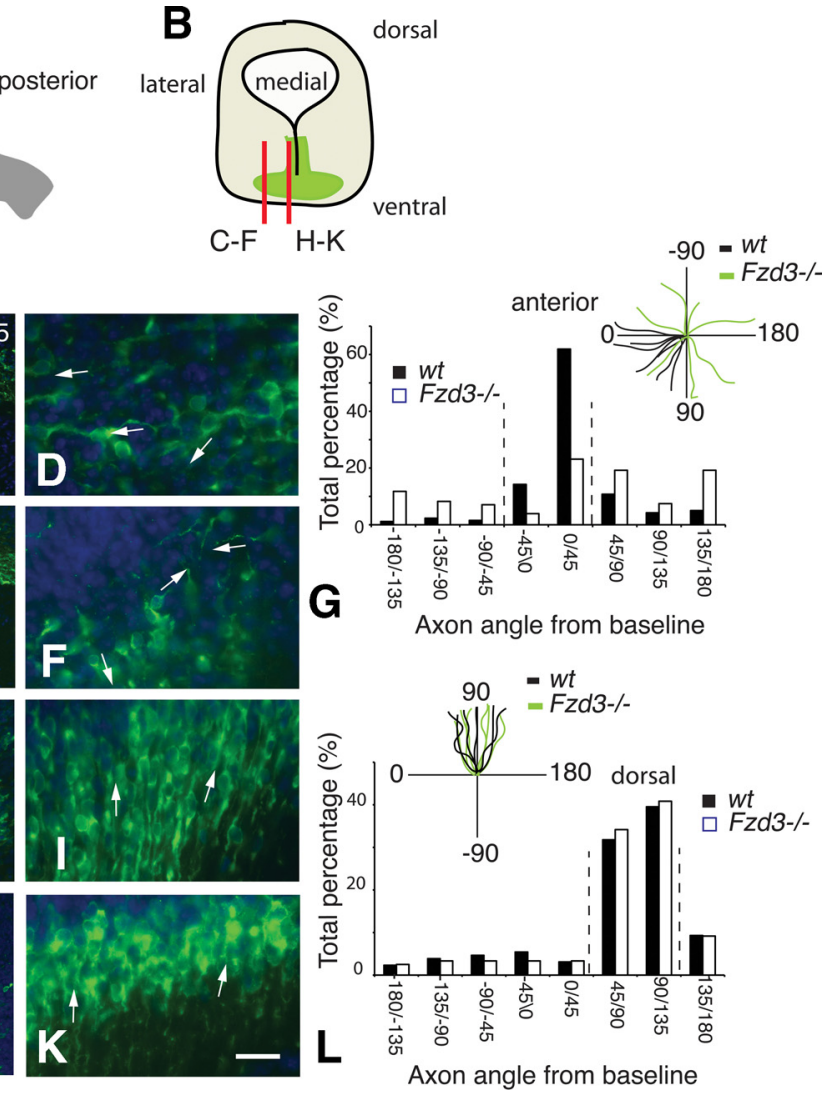

Axon angle from baseline

Figure 5. mdDA cell body orientation defects in Fzd3 mutant mice. $\boldsymbol{A}, \boldsymbol{B}$, Sagittal and coronal view of the mdDA system (green). $\boldsymbol{B}$, Red lines indicate the location of $\boldsymbol{C}-\boldsymbol{F}$ and $\boldsymbol{H}-\boldsymbol{K} . \mathbf{C}-\boldsymbol{F}, \boldsymbol{H}-\boldsymbol{K}$, Immunohistochemistry for TH (green) of E12.5 sagittal sections. (nt) mice, most TH-positive neurons in the lateral mdDA system project $\mathrm{Fzd}^{-/-}$mutants. This is quantified as shown in $\boldsymbol{L}$. Quantifications shown in $\boldsymbol{G}$ and $\boldsymbol{L}$ represent angles between the lining of the mesencephalic flexure (baseline represents $0^{\circ}$ ) and the trajectory of the axon segment just proximal to the cell body. Insets show axon orientation plots for a few randomly selected axons. Scale bar: $\boldsymbol{C}, \boldsymbol{E}, \boldsymbol{H}, \boldsymbol{J}, 25 \mu \mathrm{m} ; \boldsymbol{D}, \boldsymbol{F}, \boldsymbol{I}, \boldsymbol{K}, 12.5 \mu \mathrm{m}$.

The Looptail mouse carries a mutation in the Vangl2 protein that greatly affects Vangl2 stability and displays gross PCP defects (Montcouquiol et al., 2006). At E12.5, the $L p$ mouse displays axon guidance defects in both the ascending and descending populations (Figs. 2D, I, 3C). The ascending population displays a premature invasion of $\mathrm{R} 4$ and posterior projections (Fig. 2D,F,I). The descending population displays a striking over-fasciculation defect that is not present in the other PCP mutants we analyzed. Many of these axons grow together in tight bundles as if they were attracted to each other and appear much shorter (Fig. 2D). Higher-magnification images further reveal that these axons appear highly fasciculated (Fig. $3 F, G$ ). Like Fzd3 mutants, very few axons project posteriorly into the cervical spinal cord in $L p$ mice.

We next examined 5-HT axon projections in Celsr $3^{-1-}$ mice (Tissir et al., 2005). Like the Fzd3 $3^{-/-}$mouse, ascending axons in $\mathrm{Celsr}^{-1-}$ mice misproject both posteriorly and laterally (Fig. $2 \mathrm{E}$, arrowhead). Axons also invade the R4 region at E12.5, although not as prominently as in Fzd3 mutants (Fig. 2E,J). Although the descending population appears to show some orientation defects as observed in the $\mathrm{Fzd}^{-/-}$mouse, many neurons form normal descending projections (Fig. 2E). This is in sharp contrast to the $F z d 3^{-/-}$mouse, in which very few descending axons are formed at this stage.

At higher magnifications, we found that the cell bodies of wild-type neurons in the descending population are first oriented 


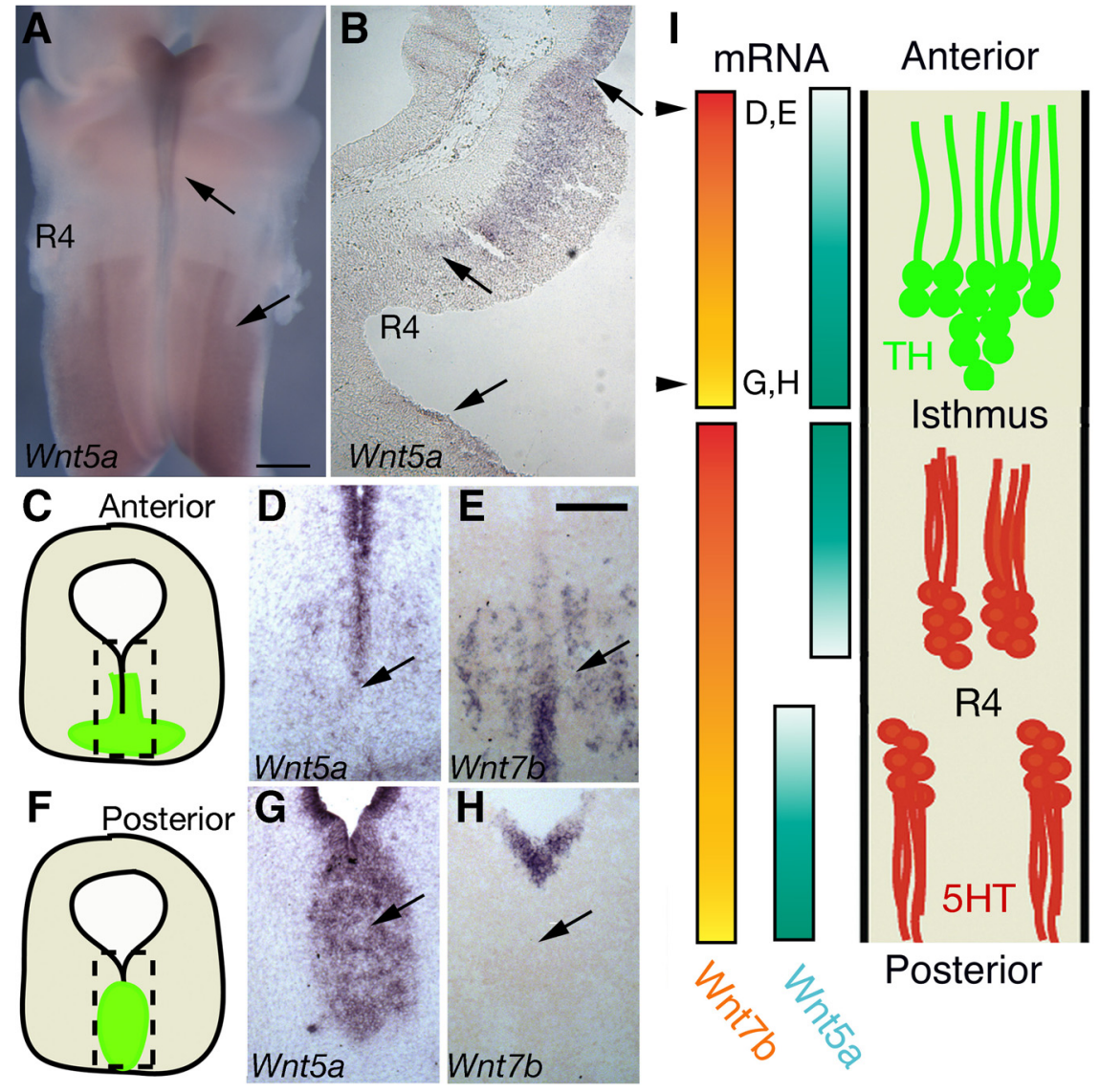

Figure 6. Multiple Wnts are expressed in gradients in both the hindbrain and midbrain. $A$, Whole-mount in situ hybridization for Wnt5a in E12.5 mouse embryos. Arrows mark the locale of both ascending and descending 5-HT populations. B, In situ hybridization for Wnt5a in a sagittal section of E12.5 hindbrain showing a anterior high, posterior low gradient near the midline and below the isthmus, consistent with the location of pathfinding ascending 5-HT axons at E12.5. C, F, Schematics indicating the location of images shown in $\mathbf{D}-\boldsymbol{H}$. In situ hybridization for Wnt5a and Wnt7b in coronal $(\boldsymbol{D}-\boldsymbol{H})$ cryosections of the E14.5 midbrain region reveals a high posterior, low anterior expression gradient for Wnt5a and a high anterior, low posterior gradient for Wnt $7 b$. I, Proposed model for the role of Wnt/PCP signaling during A-P guidance of 5-HT and TH axons. 5-HT neurons (red) express the PCP components $\mathrm{Fzd} 3$, Celsr3, and Vangl2 to sense an attractive Wnt5a expression gradient in the hindbrain region directing ascending 5-HT axons anteriorly and descending 5-HT axons posteriorly. TH neurons (green) sense both an attractive Wnt $7 \mathrm{~b}$ gradient and repulsive Wnt5a gradient guiding their axons anteriorly. Scale bars: $\boldsymbol{A}, 500 \mu \mathrm{m} ; \boldsymbol{E}, 150 \mu \mathrm{m}$.

laterally while migrating, following the direction of their leading axons but eventually becoming oriented along the A-P axis as their axons turn posteriorly (Fig. $3 \mathrm{H}-J, N$ ). However, in $\mathrm{Fzd} 3^{-1-}$ mice, many of the descending, but not ascending, cell bodies appear to remain oriented laterally or even slightly anteriorly. These cell bodies give rise to axons that grow out laterally before turning anterior or posterior (Fig. $3 K, L, O$ ). At E12.5, almost $50 \%$ of descending 5 -HT neurons in $\mathrm{Fzd}^{+/+}$mice are oriented at $180^{\circ}$ compared with $10 \%$ in $F z d 3^{-1-}$ mice (Fig. $3 P$ ). Because during normal development axons are first oriented along the $\mathrm{A}-\mathrm{P}$ axis before the soma rotates into its $\mathrm{A}-\mathrm{P}$ position, it is plausible that the cell body follows the axon and uses it to position itself along the A-P axis (Hawthorne et al., 2010). Although our observations strongly support this model, we currently cannot exclude the possibility that PCP signaling may also regulate soma and dendrite orientation independent from A-P axon guidance.

Analysis of mice deficient for Ryk (Halford et al., 2000), another Wnt receptor, did not reveal obvious defects (supplemental Fig. S2, available at www.jneurosci.org as supplemental material). Similarly, genetic inactivation of Wnt5a (Yamaguchi et al., 1999), a known PCP Wnt, also did not appear to have a striking effect on 5-HT A-P axon guidance (supplemental Fig. S2, available at www.jneurosci.org as supplemental material).

\section{PCP signaling is required for $\mathrm{A}-\mathrm{P}$ guidance of dopaminergic axons}

To assess whether Wnt/PCP signaling also controls mdDA axon patterning, we visualized mdDA axon trajectories in sections from $\mathrm{Fzd} \mathrm{3}^{-1-}$ mice using $\mathrm{TH}$ immunohistochemistry. At E11.5, many mdDA axons in $\mathrm{Fzd3}^{-/-}$mice follow an abnormal lateral trajectory at the level of the mdDA neuron pool (supplemental Fig. S3, available at www.jneurosci.org as supplemental material). At E12.5 and E14.5, a large number of mdDA axons are directed posteriorly in $\mathrm{Fzd3}^{-/-}$mice and aberrantly traverse the hindbrain (Fig. $4 A-D$ ). Although a subset of mdDA axons still projects anteriorly, presumably because of the presence of other A-P signals (Kolk et al., 2009; Yamauchi et al., 2009), this anterior projection is significantly reduced and disorganized in $\mathrm{Fzd} 3^{-/-}$mice. In addition, many mdDA axons enter abnormal dorsal territories in the midbrain and diencephalon and aberrantly approach the midline (Fig. $4 E-G$ ). In the telencephalon, mdDA axons are displaced ventrally and are located in close proximity to the optic chiasm (Fig. 4H,I). No innervation of synaptic target regions such as the striatum or medial prefrontal cortex by mdDA axons is found at E14.5 or E17.5 (Fig. $4 J, K$ ) (data not shown).

Analysis of Celsr $3^{-/-}$and Lp mice reveals similar abnormal posterior mdDA projections in the hindbrain at E12.5 and E17.5. At E12.5, Fzd3 ${ }^{-1-}$ and $L p$ mice show a similar, large number of posterior mdDA projections, whereas at this stage this defect is less pronounced in Celsr $3^{-/-}$mice (supplemental Fig. S4, available at www.jneurosci.org as supplemental material). At E17.5, however, numerous mdDA axons are projecting into the hindbrain of $\mathrm{Fzd3}^{-/-}$and $\mathrm{Celsr} 3^{-/-}$mice, but fewer posterior projections are present in $L p$ mice (Fig. $4 L-O$ ). In the diencephalon and telencephalon of Celsr $3^{-/-}$mice, many mdDA axons follow aberrant lateral, dorsal, and ventral trajectories, as observed in $\mathrm{Fzd3}^{-1-}$ mice. In contrast to $\mathrm{Fzd} 3^{-/-}$mice, however, a small subset of mdDA axons reaches, but does not innervate, the striatum in E17.5 Celsr $^{-/-}$mice (supplemental Fig. S5 A, B, available at www.jneurosci.org as supplemental material). mdDA axon projections in the forebrain of E17.5 Lp mice appear to innervate the striatum. However, it should be noted that $L p$ mice have an open neural tube and that the overall organization of the telencephalon is markedly changed in these mice (data not shown).

We also examined mdDA axon projections in $\mathrm{Wnt} 5 \mathrm{a}^{-1-}$ mice. In contrast to $\mathrm{Fzd}^{-/-}, \mathrm{Celsr} 3^{-/-}$, and $\mathrm{Lp}$ mice, $\mathrm{Wnt} 5 \mathrm{a}^{-/-}$ mice only display a minor and transient posterior projection of mdDA axons (Fig. $4 P$, inset). This suggests that the lack of Wnt5a signaling is compensated by other Wnt proteins at later developmental stages. We also analyzed mice deficient for the Wnt5a 
receptor Ryk. $R y k^{-1-}$ mice did not show overt A-P guidance defects (Fig. 4Q). In addition, mdDA innervation of the striatum appears grossly normal at E17.5 in both $W n t 5 a^{-/-}$and $R y k^{-/-}$mice (supplemental Fig. S5C-F, available at www. jneurosci.org as supplemental material).

The descending 5-HT system shows defects in cell body orientation in $\mathrm{Fzd3}^{-/-}$ embryos, which may be secondary to defective axon guidance (Fig. 3). Intriguingly, analysis of mdDA neurons at E11.5E14.5 reveals similar defects. At E11.5, the overall cellular organization of the mdDA system is normal in Fzd3 mutants (supplemental Fig. S3, available at www. jneurosci.org as supplemental material). At E12.5, mdDA cell bodies and axons in the most lateral aspect of the mdDA neuron pool of wild-type mice are oriented anteriorly (Fig. 5A-D,G). In contrast, mdDA cell bodies at the same anatomical location in $\mathrm{Fzd}^{-/-}$mice are oriented in random directions following where the axons are oriented (Fig. $5 E-G$ ). mdDA neurons in the medial part of the mdDA system of wild-type mice send their axons dorsally before these axons are redirected anteriorly (Nakamura et al., 2000) (Fig. $5 H, I, L)$. In contrast to lateral mdDA neurons, mdDA neurons in the medial part of the mdDA neuron pool in $F z d 3^{-/-}$mice do not display obvious defects in cell body orientation (Fig. 5J-L). This difference between the lateral and medial mdDA neuron pool is reminiscent of what is observed for 5-HT neurons, with descending (lateral) but not ascending (medial) neurons showing cell body orientation defects (Fig. 3).

\section{Wnts are expressed in anterior-posterior gradients in the basal midbrain and hindbrain} The only known ligands for PCP signaling are Wnt proteins. Using in situ hybridization, we sought to determine whether Wnts are expressed in the hindbrain and midbrain regions. Several Wnts were expressed in the developing hindbrain, but Wnt5a displayed the most striking expression pattern. In the hindbrain, we found that Wnt5a is expressed in an anterior high, posterior low expression pattern at the midline, where ascending serotonergic neurons are projecting anteriorly (Fig. $6 A, B$ ). Interestingly, the $W n t 5 a$ gradient direction switched from decreasing in the posterior direction to increasing at rhombomere 4 (Fig. 6 A,I). It is therefore possible that Wnt5a attraction contributes to the proper A-P guidance of both ascending and descending 5-HT axons. In the midbrain of E12.5 and E14.5 embryos, we identified clear expression gradients for Wnt5a and Wnt7b. Wnt5a labels the medial part of the mdDA system (VTA) and displays a high posterior, low anterior expression gradient, with highest expression around the isthmus (Fig. 6C, D, F,G) (Andersson et al., 2008). This Wnt5a gradient flips at the isthmus and starts to decrease toward more posterior regions (Fig. 6I). Wnt7b is expressed in both the medial and lateral mdDA system and shows a high anterior, low posterior expression pattern in the midbrain (Fig. $6 E, H$ ). In all, these experiments show that Wnt proteins form expression gradients in the hindbrain and midbrain at the time 5-HT and mdDA axons are guided into the appropriate A-P direction.

\section{Wnt proteins influence the orientation of 5-HT and mdDA axons and cell bodies}

Because Wnts are expressed in gradients in the midbrain/hindbrain region, we tested whether Wnt proteins can direct the growth of 5-HT and mdDA axons. To determine whether Wnt ligands can direct 5-HT axons, we established a new hindbrain "open-book" explant culture. We added Wnt-coated beads along the midline to disrupt endogenous Wnt gradients (Fig. 7A). The exogenous presence of Wnt 4 , Wnt5a, and Wnt7a all affected the ability of axons in the ascending population to grow anteriorly 

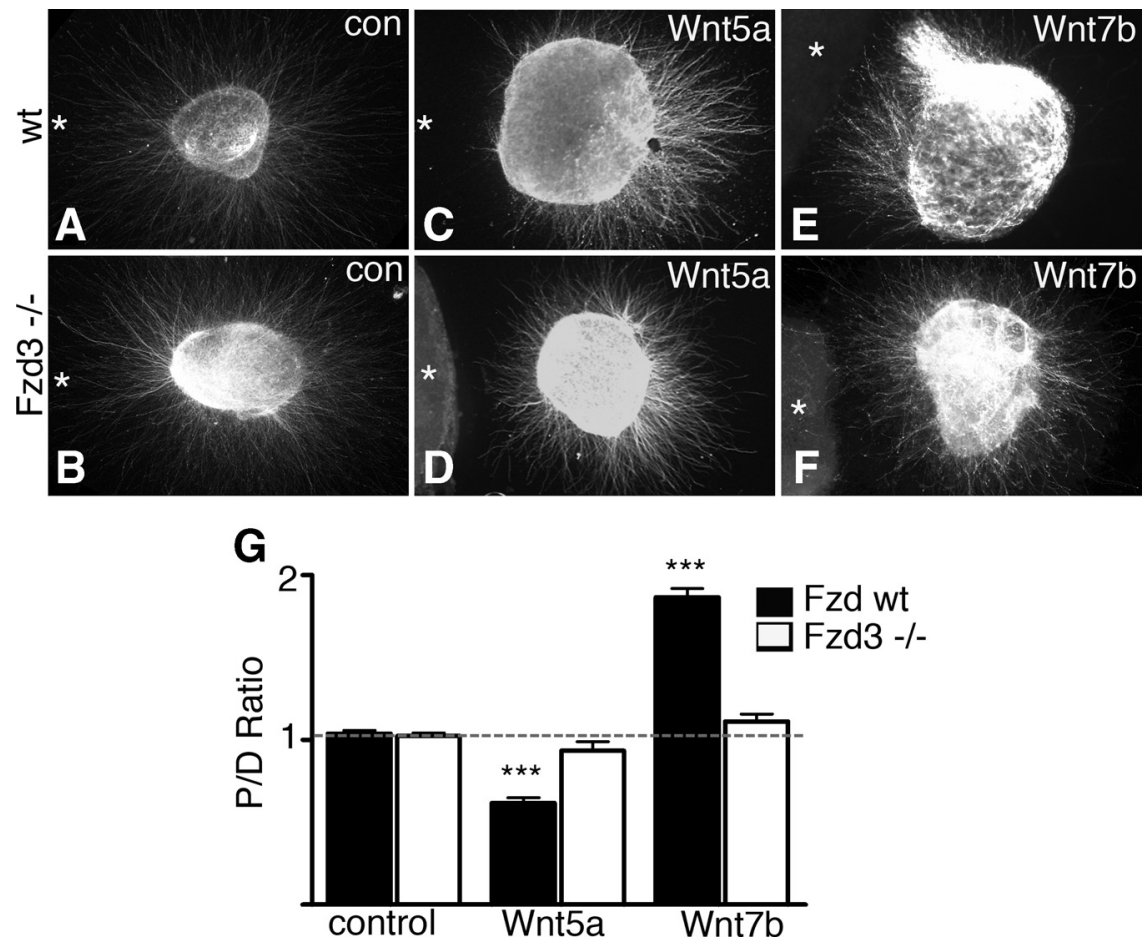

Figure 8. Dopaminergic neurons from Fzd3 mutant mice are unresponsive to Wnt5a and Wnt7b. E12.5-E14.5 VTA explants from either wild-type $(\boldsymbol{A}, \boldsymbol{C}, \boldsymbol{E})$ or Frizzled 3 mutant $(\boldsymbol{B}, \boldsymbol{D}, \boldsymbol{F})$ mice were cultured adjacent to HEK293 cells (asterisks) secreting either control (con) protein $[A, B, n=19$ for wild-type (wt) and knock-outs; Wnt5a, $C, D, n=16$ for wild-type and 18 for knock-outs; Wnt7b, $\boldsymbol{E}, \boldsymbol{F}, n=17$ for wild-type and 18 for knock-outs]. Explants are immunostained for TH. G, Quantification of the length of TH-positive neurites in the proximal and distal quadrants of culture assays. Graph shows average \pm SEM P/D ratio. Dopaminergic axons from wild-type but not $F z d 3$ mutant mice are repelled by Wnt $5 \mathrm{a}$ and attracted by Wnt $7 \mathrm{~b}$. For each experimental condition, at least three independent experiments were performed. Number of mice analyzed: $\mathrm{Fzd}^{-{ }^{-/}}, n=6 ; \mathrm{Fzd3}^{+/+}, n=8$. Scale bar, $80 \mu \mathrm{m} .{ }^{* * *} p<0.001$ compared with control, one-way ANOVA. No statistically significant differences were found between knock-out Wnt5a and wild-type control or knock-out control or between knock-out Wnt7b and wild-type control or knock-out control.

toward the brain (Fig. $7 B-D$ ). Many of these axons now grew into the midline, posteriorly, or laterally, and fewer axons projected anteriorly (see blue misprojected axons in Fig. 7D). Some cultures also displayed 5-HT axons and cell bodies that were attracted to the midline after the addition of Wnt-coated beads (Fig. 7D). These defects correlate well with the misguidance phenotypes of ascending 5-HT neurons in PCP mutant mice. The placement of the Wnt-coated beads did not appear to have a robust effect on the descending population probably because of that fact that the descending axons and cell bodies are too far away from the midline, the site of bead implantation. These experiments suggest that $\mathrm{Wnt} 5$ a serves as an attractive guidance cue for ascending 5-HT axons (Fig. 6I). To determine whether Wnts instruct mdDA axons to grow anteriorly, we used a previously established collagen matrix culture approach (Kolk et al., 2009). The chemotropic response of mdDA axons emanating from E12.5 SN or VTA explants to different Wnt proteins was tested. When confronted with cell aggregates releasing Wnt5a, THpositive SN and VTA axons displayed strong chemorepulsive responses (Fig. $7 F, I)$. In contrast, Wnt7b induced axon attraction of both mdDA axon populations (Fig. 7G,I). When examining all axons emanating from SN and VTA explants, as visualized by $\beta$ III-tubulin staining, no chemotropic effects were found, confirming the specificity of the response of TH-positive axons to Wnt5a and Wnt7b (Fig. 7H). These results together with the Wnt expression analysis suggest that Wnt5a functions to repel and
Wnt7b to attract mdDA axons into their normal anterior direction in the midbrain (Fig. 6I).

Dopaminergic axons from $\mathrm{Fzd} 3$ mutant mice are unresponsive to Wnts in vitro Our results show expression of core PCP components in 5-HT and mdDA neurons and axon projections (Fig. 1) and reveal A-P guidance defects in 5-HT and mdDA axon pathways in PCP mutant mice in vivo (Figs. 2-4). The only known PCP ligands, Wnt proteins, are expressed in instructive gradients in the brainstem and provide directional cues for 5-HT and mdDA axons in vitro (Figs. 6, 7). Together, these data support the idea that PCP proteins serve as Wnt receptors on monoaminergic axons during A-P guidance. To further test this model, we performed collagen matrix assays with VTA explants dissected from $F z d 3$ wild-type or mutant embryos. Fzd3 had been implicated as a neuronal receptor for Wnt3a and Wnt4 (Lyuksyutova et al., 2003; Endo et al., 2008). As described above, dopaminergic axons from wild-type explants were repelled by $\mathrm{Wnt} 5 \mathrm{a}$ and attracted by Wnt7b (Fig. $8 A, C, E, G$ ). In contrast, axons emanating from dopaminergic explants obtained from $\mathrm{Fzd} 3^{-/-}$mice did not display significant repulsive or attractive responses to Wnt5a and Wnt7b, respectively, compared with wild-type and knock-out control cultures (Fig. 8B,D,F,G). These results show that Fzd3 is an obligatory component of the receptor for Wnt5a and Wnt7b on dopaminergic axons and support the model that PCP receptors on monoaminergic axon projections function to sense instructive Wnt gradients in the brainstem, thereby controlling A-P axon guidance.

\section{Discussion}

Serotonergic and dopaminergic axon pathways mediate complex physiological functions and are therapeutic targets for many nervous system disorders. Their organization is exquisitely complex and requires a myriad of molecular signals for regulating different developmental steps. A crucial step in establishing monoaminergic connectivity is the initial orientation of 5-HT and mdDA axon projections along the A-P axis of the brainstem. Here we reveal a novel requirement for Wnt and PCP receptor proteins in the A-P guidance of 5-HT and mdDA axon projections. In PCP signaling mutants, both 5-HT and mdDA axons are severely misguided along the A-P axis (Fig. 9). Furthermore, we show that A-P cellular organization (i.e., cell body orientation) of monoaminergic nuclei is affected in Wnt/PCP mutants, without marked defects in the patterning of the brainstem (supplemental Fig. S6, available at www.jneurosci.org as supplemental material).

\section{Wnt signaling controls $\mathrm{A}-\mathrm{P}$ axon guidance beyond the spinal cord}

Wnt family proteins have been identified as guidance cues that pattern axonal connections along the A-P axis in vertebrates 
and invertebrates (Zou, 2006; Zou and Lyuksyutova, 2007; Salinas and Zou, 2008). In vertebrate species, Wnts provide directional cues for ascending sensory and descending corticospinal axons along the A-P axis of the spinal cord (Lyuksyutova et al., 2003; Liu et al., 2005; Wolf et al., 2008). Until now, it was unknown whether A-P guidance in parts of the CNS outside the spinal cord also required Wnt signaling. In the present study, we show that indeed A-P guidance of 5-HT and mdDA axons depends on Wnt signaling. Both 5-HT and mdDA neurons and axons expressed PCP components (Fig. 1), and mice lacking these proteins displayed prominent $\mathrm{A}-\mathrm{P}$ guidance defects in the midbrain and hindbrain (Figs. 2-4, 9). The only known PCP receptor ligands, Wnt proteins, were expressed in gradients in the brainstem and mediated guidance responses from 5-HT and mdDA axons in culture (Figs. 6, 7). mdDA neurons lacking the PCP receptor Fzd3 did not show significant axonal responses to Wnt5a and Wnt7b in culture (Fig. 8). These results establish that Wnt signaling through PCP pathway is a global A-P guidance mechanism that patterns axon tracts both in and outside the spinal cord. The marked defects in mdDA axon projections found in the telencephalon of Fzd3 and Celsr3 mutant mice (Fig. 4) support the idea that Wnt signaling may also control A-P guidance in the forebrain. In future studies, it will therefore be interesting to explore whether mdDA pathways or other longitudinal axon bundles in the forebrain are organized along the A-P axis by Wnt signaling.

\section{Complexity of Wnt signaling during A-P guidance in the brainstem}

Our study also reveals the remarkable complexity of Wnt signaling in the brainstem. Wnt gradients in the brainstem are not unidirectional, and the same Wnt gene (e.g., Wnt5a) can display opposite gradients anterior or posterior to morphologically recognizable boundary structures, such as the isthmus, or morphologically nonrecognizable but molecular defined structures, such as rhombomere 4 (Fig. 6). Furthermore, the same mdDA neurons can respond differently to different Wnts, which are expressed in opposite gradients in the midbrain (attraction by Wnt7b and repulsion by Wnt5a) to ensure correct A-P guidance (Fig. 7). In addition, neighboring neuronal populations (mdDA and 5-HT) respond differentially to the same Wnt protein (Wnt5a repels mdDA axons but attracts 5-HT axons) (Fig. 7). The molecular mechanism that underlies this difference in Wnt5a responsiveness is currently unknown but is likely to involve additional receptor proteins.

There may also be redundancy in the PCP signaling components involved in 5-HT and mdDA A-P guidance. Fzd3 mutants show broad and strong defects. In contrast, mutations in Celsr 3 and Vangl2 result in less severe or partial phenotypes. For example, descending 5-HT neurons show much weaker phenotypes in axon guidance and cell body defects in $\mathrm{Celsr} 3^{-/-}$compared with
$\mathrm{Fzd3}^{-1-}$ mice (Fig. 2). Potentially, other members of the Celsr family, Celsr1 or Celsr2 (Tissir et al., 2002), compensate for the loss of function of Celsr3. The persistence of a small number of appropriate 5-HT and mdDA A-P projections in different PCP mutants further indicates that, in addition to PCP signals, other molecular cues are involved in A-P guidance in the brainstem (Kolk et al., 2009; Yamauchi et al., 2009). Finally, Looptail mice display a severe hyperfasciculation phenotype that is not found in $\mathrm{Fzd}^{-1-}$ and $\mathrm{Celsr} 3^{-1-}$ mice, suggesting that Vangl2 may also control axon-axon interactions (Fig. 3).

Although there is a transient defect in mdDA axon projections in $W n t 5 a^{-/-}$embryos, we did not observe a similar phenotype in 5-HT axon projections. In addition to Wnt5a, Wnt4 and $W n t 7 b$ are expressed in the floor plate. The Wnt7b and Wnt5a gradients follow the same direction at the level of the ascending 5-HT system, and both Wnts appear to attract ascending 5-HT axons. Thus, Wnt7b may compensate for the loss of Wnt5a (Fig. 6). It is interesting that, although Wnt7b may attract mdDA axons anteriorly, we still observed a transient TH axon phenotype in the absence of Wnt5a (Fig. 4). It is possible that mdDA axons depend more on Wnt5a repulsion than Wnt7b attraction for anterior growth or perhaps the Wnt5a gradient is established first to repel mdDA axons anteriorly, after which the Wnt7b gradient is formed slightly later to further attract mdDA axons anteriorly (Fig. 6). In this model, mdDA axon projections may eventually become corrected by the Wnt7b gradient in Wnt5a $a^{-/-}$mice. Alternatively, aberrant axons in $W n t 5 a^{-1-}$ mice may not survive and die back at later developmental stages. Future studies will address these and other possible mechanisms. 


\section{Wnt/PCP signaling dictates cell body orientation along the A-P axis}

Neural circuit construction involves the proper orientation of axons, dendrites, and cell bodies. Because of their well documented anatomical organization, dopaminergic and serotonergic nuclei comprise an advantageous system for studying molecular cues that establish the polarity of neurons and their projections during development (Rubenstein, 1998; Goridis and Rohrer, 2002; Cordes, 2005; Smidt and Burbach, 2007; Van den Heuvel and Pasterkamp, 2008). In this study, we report that Wnt signaling is required for two different aspects of monoaminergic circuit organization. First, Wnt signaling controls the A-P guidance of monoaminergic axons. Second, proper Wnt signaling is also required for the correct cell body orientation of both 5-HT and mdDA neurons in the brainstem, which is disrupted in Fzd3 mutant mice. We found that, at least for 5-HT neurons, axon guidance appears to precede the final orientation of the soma. In wild-type animals, cell bodies of migrating, descending 5-HT neurons initially point laterally but begin to reorient along the $\mathrm{A}-\mathrm{P}$ axis as they approach their final lateral position, thereby obeying the direction of their axon projections (Fig. $3 N$ ). Recent work by Hawthorne et al. (2010) uncovers similar mechanisms and indicates that early-differentiated 5-HT neurons migrate ventrally from the ventricular zone to the pial surface by somal translocation. During somal translocation, neurons maintain a bipolar morphology, and the soma translocates along primitive ventricular and pial processes. The lateral migration of descending 5-HT neurons observed in our open-book preparations is reminiscent of somal translocation, with the axon being the leading process through which the cell body translocates. This would suggest that the direction of the axon projections dictate cell body orientation. However, one cannot exclude the possibility that cell body orientation and thus dendrite orientation may also be regulated independently from the axons by Wnt/PCP signaling. Future studies combining time-lapse imaging (Hawthorne et al., 2010), fluorescently labeled mouse lines [e.g., ePet-EYFP lines (Scott et al., 2005)], and our newly developed open-book cultures will be required to further unveil the specific mechanisms underlying cell body reorientation.

mdDA neurons are born in the ventricular zone of the mesodiencephalon and migrate ventrally along the processes of radial glia to ultimately form the SN and VTA (Smidt and Burbach, 2007; Tang et al., 2009). In PCP mutants, ventral (radial) migration of mdDA neurons is intact, but, in the most distal part of this migratory process, the orientation of mdDA cell bodies that will eventually occupy more lateral domains (e.g., the $\mathrm{SN}$ ) is perturbed (data not shown). One model to explain these defects, based on our observations in the descending 5 -HT system, is that the final stage of mdDA neuron migration, but not the radial migration, is guided by PCP signaling. Similar to what we suggest for the more laterally located descending 5-HT neurons, PCP signaling may indirectly influence mdDA neuron cell body orientation by controlling axon guidance. Future experiments are needed to address these and other possibilities.

\section{Concluding remarks}

Here we show that Wnt/PCP signaling determines the proper A-P organization of ascending and descending serotonergic and dopaminergic pathways in the brainstem. Although Wnts had been reported previously to regulate the generation and differentiation of mdDA neurons (Andersson et al., 2008), our work establishes that these proteins are reused as guidance cues for monoaminergic axons at subsequent developmental stages. This finding unveils a previously poorly characterized aspect of mdDA pathway formation and identifies one of the first guidance mechanisms involved in 5-HT circuitry formation in vivo. Furthermore, this is, to our knowledge, the first example of a role for Wnt/PCP signaling in A-P axon guidance outside the spinal cord.

\section{References}

Andersson ER, Prakash N, Cajanek L, Minina E, Bryja V, Bryjova L, Yamaguchi TP, Hall AC, Wurst W, Arenas E (2008) Wnt5a regulates ventral midbrain morphogenesis and the development of A9-A10 dopaminergic cells in vivo. PLoS ONE 3:e3517.

Cordes SP (2005) Molecular genetics of the early development of hindbrain serotonergic neurons. Clin Genet 68:487-494.

Endo Y, Beauchamp E, Woods D, Taylor WG, Toretsky JA, Uren A, Rubin JS (2008) Wnt-3a and Dickkopf-1 stimulate neurite outgrowth in Ewing tumor cells via a Frizzled3- and c-Jun N-terminal kinase-dependent mechanism. Mol Cell Biol 28:2368-2379.

Goodrich LV (2008) The plane facts of PCP in the CNS. Neuron 60:9-16.

Goridis C, Rohrer H (2002) Specification of catecholaminergic and serotonergic neurons. Nat Rev Neurosci 3:531-541.

Halford MM, Armes J, Buchert M, Meskenaite V, Grail D, Hibbs ML, Wilks AF, Farlie PG, Newgreen DF, Hovens CM, Stacker SA (2000) Rykdeficient mice exhibit craniofacial defects associated with perturbed Eph receptor crosstalk. Nat Genet 25:414-418.

Hawthorne AL, Wylie CJ, Landmesser LT, Deneris ES, Silver J (2010) Serotonergic neurons migrate radially through the neuroepithelium by dynamin-mediated somal translocation. J Neurosci 30:420-430.

Henrique D, Adam J, Myat A, Chitnis A, Lewis J, Ish-Horowicz D (1995) Expression of a Delta homologue in prospective neurons in the chick. Nature 375:787-790.

Kolk SM, Gunput RA, Tran TS, van den Heuvel DM, Prasad AA, Hellemons AJ, Adolfs Y, Ginty DD, Kolodkin AL, Burbach JP, Smidt MP, Pasterkamp RJ (2009) Semaphorin 3F is a bifunctional guidance cue for dopaminergic axons and controls their fasciculation, channeling, rostral growth, and intracortical targeting. J Neurosci 29:12542-12557.

Liu Y, Shi J, Lu CC, Wang ZB, Lyuksyutova AI, Song X, Zou Y (2005) Rykmediated Wnt repulsion regulates posterior-directed growth of corticospinal tract. Nat Neurosci 8:1151-1159.

Lyuksyutova AI, Lu CC, Milanesio N, King LA, Guo N, Wang Y, Nathans J, Tessier-Lavigne M, Zou Y (2003) Anterior-posterior guidance of commissural axons by Wnt-frizzled signaling. Science 302:1984-1988.

Montcouquiol M, Sans N, Huss D, Kach J, Dickman JD, Forge A, Rachel RA, Copeland NG, Jenkins NA, Bogani D, Murdoch J, Warchol ME, Wenthold RJ, Kelley MW (2006) Asymmetric localization of Vangl2 and Fz3 indicate novel mechanisms for planar cell polarity in mammals. J Neurosci 26:5265-5275.

Nakamura S, Ito Y, Shirasaki R, Murakami F (2000) Local directional cues control growth polarity of dopaminergic axons along the rostrocaudal axis. J Neurosci 20:4112-4119.

Pardo CA, Eberhart CG (2007) The neurobiology of autism. Brain Pathol 17:434-447.

Pasterkamp RJ, Kolk SM, Hellemons AJ, Kolodkin AL (2007) Expression patterns of semaphorin7A and plexinC1 during rat neural development suggest roles in axon guidance and neuronal migration. BMC Dev Biol 7:98.

Rubenstein JL (1998) Development of serotonergic neurons and their projections. Biol Psychiatry 44:145-150.

Salinas PC, Zou Y (2008) Wnt signaling in neural circuit assembly. Annu Rev Neurosci 31:339-358.

Scott MM, Wylie CJ, Lerch JK, Murphy R, Lobur K, Herlitze S, Jiang W, Conlon RA, Strowbridge BW, Deneris ES (2005) A genetic approach to access serotonin neurons for in vivo and in vitro studies. Proc Natl Acad Sci U S A 102:16472-16477.

Simons M, Mlodzik M (2008) Planar cell polarity signaling: from fly development to human disease. Annu Rev Genet 42:517-540.

Smidt MP, Burbach JP (2007) How to make a mesodiencephalic dopaminergic neuron. Nat Rev Neurosci 8:21-32.

Tang M, Miyamoto Y, Huang EJ (2009) Multiple roles of beta-catenin in controlling the neurogenic niche for midbrain dopamine neurons. Development 136:2027-2038.

Tissir F, De-Backer O, Goffinet AM, Lambert de Rouvroit C (2002) Developmental expression profiles of Celsr (Flamingo) genes in the mouse. Mech Dev 112:157-160. 
Tissir F, Bar I, Jossin Y, De Backer O, Goffinet AM (2005) Protocadherin Celsr3 is crucial in axonal tract development. Nat Neurosci 8:451-457.

Van den Heuvel DM, Pasterkamp RJ (2008) Getting connected in the dopamine system. Prog Neurobiol 85:75-93.

Wang Y, Nathans J (2007) Tissue/planar cell polarity in vertebrates: new insights and new questions. Development 134:647-658.

Wang Y, Thekdi N, Smallwood PM, Macke JP, Nathans J (2002) Frizzled-3 is required for the development of major fiber tracts in the rostral CNS. J Neurosci 22:8563-8573.

Wolf AM, Lyuksyutova AI, Fenstermaker AG, Shafer B, Lo CG, Zou Y (2008) Phosphatidylinositol-3-kinase-atypical protein kinase $\mathrm{C}$ signaling is required for Wnt attraction and anterior-posterior axon guidance. J Neurosci 28:3456-3467.
Yamaguchi TP, Bradley A, McMahon AP, Jones S (1999) A Wnt5a pathway underlies outgrowth of multiple structures in the vertebrate embryo. Development 126:1211-1223.

Yamauchi K, Mizushima S, Tamada A, Yamamoto N, Takashima S, Murakami F (2009) FGF8 signaling regulates growth of midbrain dopaminergic axons by inducing semaphorin 3F. J Neurosci 29:40444055

Zallen JA (2007) Planar polarity and tissue morphogenesis. Cell 129: $1051-1063$.

Zou Y (2006) Navigating the anterior-posterior axis with wnts. Neuron 49:787-789.

Zou Y, Lyuksyutova AI (2007) Morphogens as conserved axon guidance cues. Curr Opin Neurobiol 17:22-28. 